517.5517 .9

doi:10.13108/2017-9-2-102

UDC 517.5517 .9

\title{
LOWER BOUND FOR THE HARDY CONSTANT FOR AN ARBITRARY DOMAIN IN $\mathbb{R}^{n}$
}

\section{I.K. SHAFIGULLIN}

\begin{abstract}
In the paper we consider the conjecture by E.B. Davies on an uniform lower bound for the Hardy constant. We provide the known counterexamples rebutting this conjecture for the dimension 4 and higher. In the work we obtain non-zero lower bounds for the Hardy constants. These estimates are order sharp as $n \rightarrow+\infty$, where $n$ is the space dimension. Moreover, these estimates are independent of the properties of the considered domains and are true for all domains not coinciding with the entire space. In the proof of the main theorem we reduce the multidimensional case to the one-dimensional case by choosing special classes of functions. As a result, the considered inequalities are reduced to the well-known Poincaré inequality.
\end{abstract}

Keywords: Hardy constant, lower bounds, Hardy inequalities, variational inequalities.

Mathematics Subject Classification: 26D15

\section{INTRODUCTION}

Let $\Omega \subset \mathbb{R}^{n}$ be a domain, $\Omega \neq \mathbb{R}^{n}$. By $\delta(x)$ we denote the distance to the boundary of the domain, namely,

$$
\delta(x)=\inf _{y \in \partial \Omega}|x-y|
$$

In view of the introduced definitions, we consider the Hardy type inequality

$$
\int_{\Omega} \frac{|f(x)|^{2}}{\delta^{2}(x)} d x \leqslant c_{n}(\Omega) \int_{\Omega}|\nabla f(x)|^{2} d x
$$

for all $f \in C_{0}^{1}(\Omega)$, where $C_{0}^{1}(\Omega)$ is the set of continuously differentiable compactly supported functions on $\Omega$ and $c_{n}(\Omega)$ is the smallest possible constant in this inequality for the given domain $\Omega \neq \mathbb{R}^{n}$.

By $c_{n}$ we denote the maximal constant bounding from below the Hardy constant in the above given inequality, namely,

$$
c_{n}=\inf _{\Omega \subset \mathbb{R}^{n}, \Omega \neq \mathbb{R}^{n}} c_{n}(\Omega)=\inf _{\Omega \subset \mathbb{R}^{n}, \Omega \neq \mathbb{R}^{n}} \sup _{f \in C_{0}^{1}(\Omega), f \neq 0} \frac{\int_{\Omega} \frac{|f(x)|^{2}}{\delta^{2}(x)} d x}{\int|\nabla f(x)|^{2} d x} .
$$

It is obvious that $c_{n} \geqslant 0$. Many mathematicians studied this quantity. The most well studied are one-dimensional Hardy type inequalities. For instance, it is well-known that as $n=1$, the classical results by Hardy imply that

$$
c_{1}=4
$$

I.K. Shafigullin, Lower Bound for the HARdy CONSTANt FOR AN ARBitrary DOMAin in $\mathbb{R}^{n}$.

(c) I.K. Shafigullin. 2017.

The work is supported by Russian Foundation for Basic Researches (grant no. 14-01-00751).

Submitted May 19, 2016. 
Moreover, he showed that the constant is not attained, that is, there is no extremal function $f \not \equiv 0$ turning the integral inequality into the identity with the constant $c_{1}=4$.

It should be noted that studying one-dimensional Hardy type inequalities involves much more issues apart of estimating of the quantity $c_{1}$. A valuable contribution in the developing of this subject was made by works by V.G. Maz'ya [1], G. Talenti [2], G. Tomaselli [3], A. Kufner and L.E. Persson [4], Yu.A. Dubinskii [5], D.V. Prokhorov and V.D. Stepanov [6], F.G. Avkhadiev and K.-J. Wirths [7] and by many other mathematicians. The results are obtained both for various weight functions and for inequalities with many terms. Much attention was paid to the sharpness of the constants.

The studying of one-dimensional Hardy type inequalities is still ongoing. However, in the last 30 years, much more attention was paid to their multi-dimensional analogues. The theory of multi-dimensional Hardy type inequalities contains many unsolved issues. For instance, the issue on estimating the constant $c_{n}$ for the inequalities in $n$-dimensional space as $n \geqslant 2$ is still unsolved. At present, the researchers succeeded to obtain uniform estimates for these constants for arbitrary domains. However, there were studied wide classes of the domains, for which the lower estimates for the Hardy constants are obtained. For instance, it is shown that for each convex domain we also have the inequality

$$
c_{n}(\Omega)=4 .
$$

This fact was proven by many mathematicians via using various technical tricks. One of the simplest proofs was provided in paper [8], see also [9]-[12].

Moreover, $c_{n}(\Omega)>0$ for each bounded domain with a locally Lipschitz boundary, see, for instance, [13]. However, it is unclear whether the quantity $\inf _{\Omega} c_{n}(\Omega)$ is positive even if the infimum is taken over the class of the domains with locally Lipschitz boundaries.

While studying lower estimates for the Hardy constants, E.B. Davies employed another approach, namely, he showed that the Hardy constant has a locally geometric nature and while estimating it from below, one does not need to known the global structure of the domain but it is sufficient to know only local structure of the boundary in the vicinity of one boundary point [9]. Thus, there was obtained the result that if the boundary of the domain $\Omega \subset \mathbb{R}^{n}$ has at least one point regular in the Davies sense, then the inequality

$$
c_{n}(\Omega) \geqslant 4
$$

holds true. The definition of a point regular in the Davies sense can be found in its work [9]. As a result he obtained that for a rather wide class of the domains obeying the known results, the sought constant can not be less than 4. In 1997 he proposed a conjecture that the constant $c_{n}(\Omega)$ should be at least 4 for each domain with no restrictions, as in the case $n=1$. As $n=2$ and $n=3$, there is no counterexample. But as $n \geqslant 4$, such counterexample exists, see, for instance, [7], [8]. The following inequality is known:

$$
\int_{\mathbb{R}^{n}} \frac{|f(x)|^{2}}{|x|^{2}} d x \leqslant \frac{4}{(n-2)^{2}} \int_{\mathbb{R}^{n}}|\nabla f(x)|^{2} d x
$$

for all $f \in C_{0}^{1}\left(\mathbb{R}^{n}\right)$. Since for $\Omega=\mathbb{R}^{n} \backslash\{0\}$ the distance to the boundary of the domains is expressed as $\operatorname{dist}(x, \partial \Omega)=\delta(x)=|x|$, we can rewrite this inequality as

$$
\int_{\Omega} \frac{|f(x)|^{2}}{\delta^{2}(x)} d x \leqslant \frac{4}{(n-2)^{2}} \int_{\Omega}|\nabla f(x)|^{2} d x
$$

for all $f \in C_{0}^{1}(\Omega)$. This means that as $n \geqslant 4$, the inequality

$$
c_{n} \leqslant \frac{4}{(n-2)^{2}}<4
$$


holds true. Thus, the conjecture by E.B. Davies turns out to be wrong for $n \geqslant 4$.

In the present work we consider lower bounds for the Hardy constants. Our results on the upper bounds can be found in works [14], [15], [16].

Such intent and long attention to Hardy type inequalities is explained by the fact that these inequalities have wide applications in various fields of the mathematics. For instance, they play an important role in studying problems arising in functional analysis (in theory of embedding functional spaces), operator theory (in the theory of elliptic operators and spectral theory), the theory of differential equations (in the problem on resonant states existence), theory of integral equation (in Sobolev-Hardy type inequalities), mathematical physics and nonlinear analysis.

\section{Formulation of PROBlem and main RESUlts}

In view of the introduced definitions we formulate a theorem giving a lower bound for the Hardy constant for an arbitrary domain not coinciding with the entire space.

Theorem 2.1. The constant $c_{n}$ satisfies the inequality

$$
c_{n}=\inf _{\Omega \subset \mathbb{R}^{n}, \Omega \neq \mathbb{R}^{n}} \sup _{f \in C_{0}^{1}(\Omega), f \neq 0} \frac{\int_{\Omega} \frac{|f(x)|^{2}}{\delta^{2}(x)} d x}{\int|\nabla f(x)|^{2} d x} \geqslant \frac{2 \pi^{2}}{5\left(8+n(n+1) \pi^{2}\right)} .
$$

Proof. Consider an arbitrary domain $\Omega$. Let $B(x, r)$ be an open ball of radius $r$ centered at the point $x$. The boundary of the domain $\Omega$ possesses at least one finite boundary point and this means that we can consider a ball inside the domain $\Omega$, whose boundary contains at least one point of the boundary of $\Omega$. More rigorously we write this as

$$
\exists x_{0} \in \Omega, r_{0}>0 \mid B\left(x_{0}, r_{0}\right) \subset \Omega \text { and } \partial B\left(x_{0}, r_{0}\right) \bigcap \partial \Omega \neq \emptyset .
$$

We choose arbitrarily one of the boundary point lying in the intersection of the boundary of the ball $B\left(x_{0}, r_{0}\right)$ and the domain $\Omega$. For the sake of the definiteness we denote it by $a$, that is,

$$
a \in \partial B\left(x_{0}, r_{0}\right) \bigcap \partial \Omega \text {. }
$$

It can be shown that the constant in Hardy type inequalities is invariant under conformal linear transformations and therefore, without loss of generality we can suppose that

$$
x_{0}=0, r_{0}=1, a=(1,0, \ldots, 0) \in \mathbb{R}^{n} .
$$

We pass to the spherical coordinate system:

$$
\left\{\begin{array}{l}
x_{1}=r \cos \left(\varphi_{n-1}\right) \\
x_{2}=r \sin \left(\varphi_{n-1}\right) \cos \left(\varphi_{n-2}\right) \\
\ldots \ldots \ldots \ldots \ldots \ldots \ldots \ldots \ldots \ldots \ldots \ldots \ldots \ldots \ldots \ldots \ldots \ldots \ldots \\
x_{n-1}=r \sin \left(\varphi_{n-1}\right) \sin \left(\varphi_{n-2}\right) \ldots \sin \left(\varphi_{2}\right) \cos \left(\varphi_{1}\right) \\
x_{n}=r \sin \left(\varphi_{n-1}\right) \sin \left(\varphi_{n-2}\right) \ldots \sin \left(\varphi_{2}\right) \sin \left(\varphi_{1}\right)
\end{array}\right.
$$

where

$$
r \in \mathbb{R}, r \geqslant 0 ; \varphi_{1} \in[0,2 \pi), \varphi_{k} \in[0, \pi], k=\overline{2, n-1} .
$$

The Jacobian of such transformation is

$$
r^{n-1} \sin ^{n-2}\left(\varphi_{n-1}\right) \sin ^{n-3}\left(\varphi_{n-2}\right) \ldots \sin \varphi_{2} .
$$

The introduced ball $B(0,1)$ in spherical coordinates is defined as

$$
x \in \mathbb{R}^{n} \mid r \in[0,1), \varphi_{1} \in[0,2 \pi), \varphi_{k} \in[0, \pi], k=\overline{2, n-1} .
$$

The coordinates of the point $a$ in the introduced coordinate system are

$$
r=1, \quad \varphi_{n-1}=0, \quad \varphi_{1} \in[0,2 \pi), \quad \varphi_{k} \in[0, \pi], \quad k=\overline{2, n-2} .
$$


We need to estimate the Hardy constant from below and this means that it is sufficient to prove this statement on a smaller class of functions than the initially considered class $C_{0}^{1}(\Omega)$. In order to do it, we introduce the domain

$$
A_{\alpha, \rho}=\left\{x \in \Omega \mid r \in(\rho, 1), \varphi_{n-1} \in(0, \alpha), \varphi_{1} \in[0,2 \pi), \varphi_{k} \in[0, \pi], k=\overline{2, n-2}\right\},
$$

where $\rho \in(0,1), \alpha \in\left(0, \frac{\pi}{2}\right)$. It is obvious that $A_{\alpha, \rho} \subset B \subset \Omega$.

For further studies it will be important to understand how the boundary of the domain $A_{\alpha, \rho}$ looks like. Let us write it explicitly:

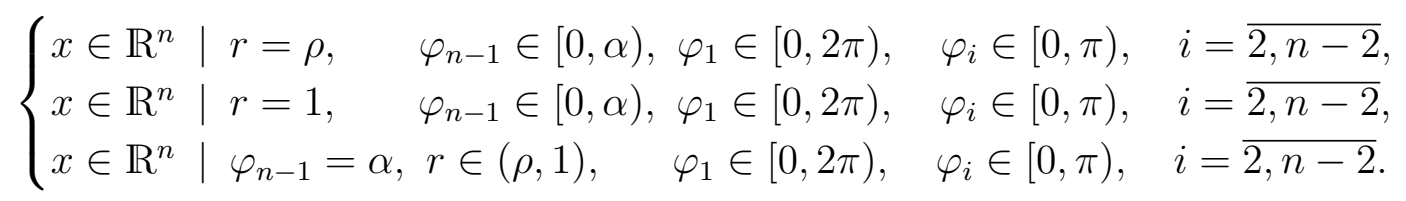

We choose domains $A_{\alpha}$ as follows: among all domains $A_{\alpha, \rho}$ we consider only ones satisfying the conditions $1-\rho=2 \alpha$ and $\alpha<\frac{\pi}{10}$. It can be shown that in $A_{\alpha} \subset \Omega$ the following inequalities

$$
\delta(x) \geqslant 1-r, \quad \delta^{2}(x) \leqslant(1-r)^{2}+\alpha^{2}
$$

hold true for each point $x \in A_{\alpha}$, where $r$ is the radius vector of the considered point and $\delta(x)$ is the distance to the boundary of the domain $\Omega$.

Now we consider functions $f \in C_{0}^{1}(\Omega)$ compactly supported in $A_{\alpha}$ and expressed as the product of two functions of one variable:

$$
f_{0}\left(r, \varphi_{1}, \ldots, \varphi_{n-1}\right)=u(r) v\left(\varphi_{n-1}\right) .
$$

To simplify the understanding, we denote the functions of such kind by $f_{0}$ and write the original inequality for such functions

$$
\int_{\Omega} \frac{\left|f_{0}(x)\right|^{2}}{\delta^{2}(x)} d x \leqslant c_{n}(\Omega) \int_{\Omega}\left|\nabla f_{0}\right|^{2} d x
$$

for all $f_{0} \in C_{0}^{1}\left(A_{\alpha}\right)$.

We pass to spherical coordinates and use the fact that the support of our function is contained in $A_{\alpha}$ :

$$
\begin{aligned}
& \int_{A_{\alpha}} \frac{\left|f_{0}\right|^{2}}{\delta^{2}} r^{n-1} \sin ^{n-2}\left(\varphi_{n-1}\right) \sin ^{n-3}\left(\varphi_{n-2}\right) \ldots \sin \varphi_{2} d r d \varphi_{1} \ldots d \varphi_{n-1} \\
& \quad \leqslant c_{n}(\Omega) \int_{A_{\alpha}}\left|\nabla f_{0}\right|^{2} r^{n-1} \sin ^{n-2}\left(\varphi_{n-1}\right) \sin ^{n-3}\left(\varphi_{n-2}\right) \ldots \sin \varphi_{2} d r d \varphi_{1} \ldots d \varphi_{n-1}
\end{aligned}
$$

for all $f_{0} \in C_{0}^{1}\left(A_{\alpha}\right)$.

For the sake of the convenience in further calculations, we move the constant $c_{n}(\Omega)$ in the left hand side. Since our function $f_{0}$ is defined as the product of two functions of one variable, we can calculate the length of the gradient of $f_{0}$ by the following formula:

$$
\left|\nabla f_{0}\right|^{2}=\left(\frac{\partial f_{0}}{\partial r}\right)^{2}+\frac{1}{r^{2}}\left(\frac{\partial f_{0}}{\partial \varphi_{n-1}}\right)^{2}
$$

We employ this relation in the previous inequality:

$$
\frac{1}{c_{n}(\Omega)} \int_{0}^{\alpha} v^{2}\left(\varphi_{n-1}\right) \sin ^{n-2}\left(\varphi_{n-1}\right) \int_{\rho}^{1} \frac{u^{2}(r) r^{n-1}}{(1-r)^{2}+\alpha^{2}} d r Q
$$




$$
\begin{aligned}
\leqslant & \int_{0}^{\alpha} v^{2}\left(\varphi_{n-1}\right) \sin ^{n-2}\left(\varphi_{n-1}\right) \int_{\rho}^{1} u^{\prime 2}(r) r^{n-1} d r Q \\
& +\int_{0}^{\alpha} v^{\prime 2}\left(\varphi_{n-1}\right) \sin ^{n-2}\left(\varphi_{n-1}\right) \int_{\rho}^{1} u^{2}(r) r^{n-3} d r Q,
\end{aligned}
$$

where

$$
Q=\int_{0}^{\pi} \sin ^{n-3}\left(\varphi_{n-2}\right) d \varphi_{n-2} \cdots \int_{0}^{\pi} \sin \left(\varphi_{2}\right) d \varphi_{2} \int_{0}^{2 \pi} d \varphi_{1}
$$

The quantity $Q$ is a positive number. We divide the inequality by this constant. We need to estimate the integral of $v^{\prime 2}(\varphi)$ by the integral of $v^{2}(\varphi)$. Then we can cancel out this integral and reduce all to the well-studied case of the integral inequality for a function of one variable. We shall make such estimate in two stages.

At the first stage we consider the function $v_{0}\left(\varphi_{n-1}\right)=\alpha-\varphi_{n-1}$. Let us estimate the fraction

$$
L(\alpha)=\frac{\int_{0}^{\alpha} v_{0}^{\prime 2}\left(\varphi_{n-1}\right) \sin ^{n-2}\left(\varphi_{n-1}\right) d \varphi_{n-1}}{\int_{0}^{\alpha} v_{0}^{2}\left(\varphi_{n-1}\right) \sin ^{n-2}\left(\varphi_{n-1}\right) d \varphi_{n-1}}
$$

that will help us to make the needed estimate in what follows.

We consider separately the nominator and the denominator of this fraction. We employ the well-known estimate for the function $\sin x$ :

$$
\frac{\sin \alpha}{\alpha} x \leqslant \sin x \leqslant x
$$

for all $x \in[0, \alpha]$.

Now we can consider the nominator of the studied fraction and estimate it from above:

$$
\int_{0}^{\alpha} v_{0}^{\prime 2}\left(\varphi_{n-1}\right) \sin ^{n-2}\left(\varphi_{n-1}\right) d \varphi_{n-1}=\int_{0}^{\alpha} \sin ^{n-2}\left(\varphi_{n-1}\right) d \varphi_{n-1} \leqslant \int_{0}^{\alpha} \varphi_{n-1}^{n-2} d \varphi_{n-1}=\frac{\alpha^{n-1}}{n-1} .
$$

The denominator is estimated from below:

$$
\begin{aligned}
\int_{0}^{\alpha} v_{0}^{2}\left(\varphi_{n-1}\right) \sin ^{n-2}\left(\varphi_{n-1}\right) d \varphi_{n-1} & =\int_{0}^{\alpha}\left(\alpha-\varphi_{n-1}\right)^{2} \sin ^{n-2}\left(\varphi_{n-1}\right) d \varphi_{n-1} \\
& \geqslant \frac{\sin ^{n-2}(\alpha)}{\alpha^{n-2}} \int_{0}^{\alpha} \varphi_{n-1}^{n-2}\left(\alpha-\varphi_{n-1}\right)^{2} d \varphi_{n-1} \\
& =\left(\frac{\sin \alpha}{\alpha}\right)^{n-2} \int_{0}^{\alpha} \alpha^{2} \varphi_{n-1}^{n-2}-2 \alpha \varphi_{n-1}^{n-1}+\varphi_{n-1}^{n} d \varphi_{n-1} \\
& =\left(\frac{\sin \alpha}{\alpha}\right)^{n-2} \frac{2 \alpha^{n+1}}{n(n+1)(n-1)} .
\end{aligned}
$$

Hence, we can estimate our function $L(\alpha)$ as follows:

$$
L(\alpha) \leqslant \frac{(n+1) n}{2 \alpha^{2}}\left(\frac{\alpha}{\sin \alpha}\right)^{n-2}=\frac{(n+1) n}{2 \alpha^{2}} K(\alpha),
$$

where $\lim _{\alpha \rightarrow 0} K(\alpha)=1$. 
We proceed to the second stage. In view of the structure of the function $v_{0}\left(\varphi_{n-1}\right)$, we can conclude that we can approximate it in the norm of $L_{2}\left(\sin ^{n-2}\left(\varphi_{n-1}\right)\right)$ by the functions $v_{1}\left(\varphi_{n-1}\right)$ vanishing in some neighbourhood of the point $a$ and whose derivatives vanish at 0 . Such functions satisfy the inequality:

$$
\int_{0}^{\alpha} v_{1}^{\prime 2} \sin ^{n-2}\left(\varphi_{n-1}\right) d \varphi_{n-1} \leqslant\left(\frac{n(n+1)}{2 \alpha^{2}} K(\alpha)+\varepsilon\right) \int_{0}^{\alpha} v_{1}^{2} \sin ^{n-2}\left(\varphi_{n-1}\right) d \varphi_{n-1} .
$$

We return back to the main idea of the proof and make the considered class of the functions smaller; instead of $f_{0}$, we shall consider the functions

$$
f_{1}\left(r, \varphi_{1}, \ldots, \varphi_{n-1}\right)=u(r) v_{1}\left(\varphi_{n-1}\right) .
$$

Then the initial inequality for the functions of form $f_{1}$ is reduced to the following onedimensional inequality:

$$
\frac{1}{c_{n}(\Omega)} \int_{\rho}^{1} \frac{u^{2}(r) r^{n-1}}{(1-r)^{2}+\alpha^{2}} d r \leqslant \int_{\rho}^{1} u^{\prime 2}(r) r^{n-1} d r+\left(\frac{n(n+1)}{2 \alpha^{2}} K(\alpha)+\varepsilon\right) \int_{\rho}^{1} u^{2}(r) r^{n-3} d r
$$

for all $u \in C_{0}^{1}(\rho, 1)$.

We employ that the variable $r$ ranges in $[\rho, 1]$ and make the change of variable $1-r=\alpha(1-t)$. As the result, we get:

$$
\frac{\rho^{n-1}}{\alpha^{2} c_{n}(\Omega)} \int_{-1}^{1} \frac{z^{2}(t)}{(1-t)^{2}+1} d t \leqslant \frac{1}{\alpha^{2}} \int_{-1}^{1} z^{\prime 2}(t) d t+\left(\frac{n(n+1)}{2 \alpha^{2}} K(\alpha)+\varepsilon\right) \int_{-1}^{1} z^{2}(t) d t
$$

for all $z \in C_{0}^{1}(-1,1)$. Now we pass to the limit as $\alpha$ tends to zero, at that, $\rho$ tends to one and hence,

$$
\frac{1}{c_{n}(\Omega)} \int_{-1}^{1} \frac{z^{2}(t)}{(1-t)^{2}+1} d t \leqslant \int_{-1}^{1} z^{\prime 2}(t) d t+\frac{n(n+1)}{2} \int_{-1}^{1} z^{2}(t) d t \quad \forall z \in C_{0}^{1}(-1,1) .
$$

We observe that in the integral in the left hand side the singularity disappears. Let us estimate the denominator in the integrand by the obvious inequality $(1-t)^{2}+1 \leqslant 5$ as $t \in[-1,1]$. We have:

$$
\left(\frac{1}{5 c_{n}(\Omega)}-\frac{n(n+1)}{2}\right) \int_{-1}^{1} z^{2}(t) d t \leqslant \int_{-1}^{1} z^{\prime 2}(t) d t
$$

for all $z \in C_{0}^{1}(-1,1)$. This is the known one-dimensional Poincaré inequality and the constant is estimated from below by $\frac{4}{\pi^{2}}$. This gives the sought estimate

$$
c_{n}(\Omega) \geqslant \frac{2 \pi^{2}}{5\left(8+n(n+1) \pi^{2}\right)} .
$$

Thus, we have obtained that the Hardy constant is estimated from below uniformly for each domain, that is,

The proof is complete.

$$
c_{n}=\inf _{\Omega \subset \mathbb{R}^{n}, \Omega \neq \mathbb{R}^{n}} c_{n}(\Omega) \geqslant \frac{2 \pi^{2}}{5\left(8+n(n+1) \pi^{2}\right)} .
$$

In conclusion the author would like to express his gratitude to his scientific supervisor, professor Farit Gabidinovich Avkhadiev for the attention to the work and valuable remarks. 


\section{BIBLIOGRAPHY}

1. V.G. Maz'ya. Sobolev Spaces. Springer-Verlag, Berlin (1985).

2. G. Talenti. Osservazione sopra una classe di disuguaglianze // Rend. Semin. mat. efis. Milano. 39, 171-185 (1969).

3. G. Tomaselli. A class of inequalities // Boll. Unione mat. ital. Ser. IV. 2, 622-631 (1969).

4. A. Kufner, L.-E. Persson. Weighted inequalities of Hardy type. World Scientific, New Jersey (2003).

5. Yu.A. Dubinskii. A Hardy-type inequality and its applications // Trudy Matem. Inst. Steklova. 269, 112-132 (2010). [Proc. Steklov Inst. Math. 269, 106-126 (2010).]

6. D.V. Prokhorov, V.D. Stepanov. On weighted Hardy inequalities in mixed norms // Trudy Matem. Inst. Steklova. 283, 155-170 (2013). [Proc. Steklov Inst. Math. 283, 149-164 (2013).]

7. F.G. Avkhadiev, K.-J. Wirths. Unified Poincaré and Hardy inequalities with sharp constants for convex domains // Z. Angew. Math. Mech. 87:8-9, 632-642 (2007).

8. M. Marcus, V.J. Mizel, Y. Pinchover. On the best constants for Hardy's inequality in $\mathbb{R}^{n} / /$ Trans. Amer. Math. Soc. 350:8, 3237-3250 (1998).

9. E.B. Davies. The Hardy constant // Quart. J. Math. Oxford. Ser. II. 46:184, 417-431 (1995).

10. T. Matskewich, P.E. Sobolevskii. The best possible constant in a generalized Hardy's inequality for convex domains in $\mathbb{R}^{n} / /$ Nonl. Anal. 28:9, 1601-1610 (1997).

11. H. Brezis, M. Marcus. Hardy's inequality revisited // Ann. Scuola Norm. Sup. Pisa Cl. Sci. (4). 25:1-2, 217-237 (1997).

12. M. Hoffmann-Ostenhof, T. Hoffmann-Ostenhof, A. Laptev. A geometrical version of Hardy's inequality // J. Funct. Anal. 189:2, 539-548 (2002).

13. V. Opic, A. Kufner. Hardy-type Inequalities. Pitman Research Notes Math. 219. Longman Scientific \& Technical, Harlow, John Wiley \& Sons, New York (1990).

14. F.G. Avkhadiev, I.K. Shafigullin. Sharp estimates of Hardy constants for domains with special boundary properties // Izv. VUZov. Matem. 2, 69-73 (2014). [Russ. Math. (Izv. VUZ. Matem.)

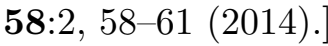

15. F.G. Avkhadiev, I.K. Shafigullin. Estimates of Hardy's constants for tubular extensions of sets and domains with finite boundary moments // Matem. Trudy. 16:2, 3-12 (2013). [Siber. Adv. Math. 24:3, 153-158 (2014).]

16. F.G. Avkhadiev, R.G. Nasibullin, I.K. Shafigullin. Hardy-type inequalities with power and logarithmic weights in domains of the Euclidean space // Izv. VUZov. Matem. 9, 90-94 (2011). [Russ. Math. Izv. VUZ. Matem. 55:9, 76-79 (2011).]

Il'nar Kasyimovich Shafigullin,

Kazan Federal University,

Kremlevskaya str., 18,

420008, Kazan, Russia

E-mail: shafigullin.ik@gmail.com 